International Journal of Pure and Applied Mathematics

Volume 92 No. 1 2014, 133-139

ISSN: 1311-8080 (printed version); ISSN: 1314-3395 (on-line version)

url: http://www.ijpam.eu

doi: http://dx.doi.org/10.12732/ijpam.v92i1.13

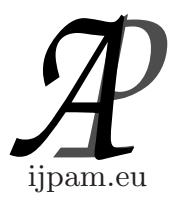

\title{
ON MAXIMAL IDEALS IN TERNARY SEMIGROUPS
}

\author{
Thawhat Changphas \\ Department of Mathematics \\ Faculty of Science \\ Khon Kaen University \\ Khon Kaen 40002, THAILAND
}

\begin{abstract}
It is known that every maximal ideals in a commutative ring with identity is prime; this result is also valid for commutative ordered semigroups and commutative semigroups as well. In this paper, we show that in commutative ternary semigroups with identity every maximal ideals is prime.
\end{abstract}

\section{AMS Subject Classification: 06F05}

Key Words: semigroup, commutative ternary semigroup with identity, ideal, prime ideal, maximal ideal, direct product

\section{Preliminaries}

It is known that the notion of ideals play an important rule in several algebraic structures such as rings, semigroups and ternary semigroups. Particularly, relations between maximal ideals and prime ideals have been widely studied by many authors. One of important results for a commutative ring with identity is every maximal ideal is a prime ideal ([1], p.128); this result is also valid for a commutative semigroup with identity [8] and also for commutative ordered semigroups with identity [4]. The purpose of this paper is to show that this result is also true for a commutative ternary semigroup with identity. We also give an example to show that the converse of the statement is not valid, in general.

Received: February 3, 2014

(c) 2014 Academic Publications, Ltd. url: www.acadpubl.eu 
Definition 1. ([5], [7]) A nonempty set $T$ with a ternary operation $(x, y, z) \mapsto[x y z]$ satisfying the associative law, that is, for any $x_{1}, x_{2}, x_{3}, x_{4}, x_{5}$ in $T$,

$$
\left[\left[x_{1} x_{2} x_{3}\right] x_{4} x_{5}\right]=\left[x_{1}\left[x_{2} x_{3} x_{4}\right] x_{5}\right]=\left[x_{1} x_{2}\left[x_{3} x_{4} x_{5}\right]\right]
$$

is called a ternary semigroup.

Note that a semigroup $S$ can be made into a ternary semigroup under the associative ternary operation defined by

$$
(x, y, z) \mapsto x y z .
$$

Definition 2. ([3]) An element $e$ of a ternary semigroup $T$ is called an identity element or unital element if

$$
[e e x]=[e x e]=[x e e]=x
$$

for all $x$ in $T$.

Definition 3. ([2]) A ternary semigroup $T$ is said to be commutative if, for all $x_{1}, x_{2}, x_{3}$ in $T$,

$$
\left[x_{1} x_{2} x_{3}\right]=\left[x_{\alpha(1)} x_{\alpha(2)} x_{\alpha(3)}\right]
$$

for all permutations $\alpha$ on $\{1,2,3\}$.

If $A, B$ and $C$ are nonempty subsets of a ternary semigroup $T$, then the product $[A B C]$ is the set of all elements $[a b c] \in T$ where $a \in A, b \in B$ and $c \in C$, i.e.,

$$
[A B C]=\{[a b c] \mid a \in A, b \in B, c \in C\} .
$$

In dealing with singleton sets we write, for example, $[\{a\} B C]$ by $[a B C]$.

Definition 4. ([6]) Let $T$ be a ternary semigroup. A nonempty subset $A$ of $T$ is called a left (respectively, middle, right) ideal of $T$ if

$$
[T T A] \subseteq A
$$

(respectively, $[T A T] \subseteq A,[A T T] \subseteq A$ ). If $A$ is both a left and a right ideal of $T$, then $A$ is called a two-sided ideal of $T$. If $A$ is a left, a middle and a right ideal of $T$, then $A$ is called an ideal of $T$.

An ideal $A$ of a ternary semigroup $T$ is said to be a proper ideal of $T$ if $A \subset T$ (The symbol $\subset$ stands for proper inclusion for sets). For proper right, left, middle and two-sided ideal are defined similarly. 
Theorem 5. ([6]) If $A$ is a nonempty subset of a ternary semigroup $T$, then

$$
\langle A\rangle=A \cup[T T A] \cup[A T T] \cup[T A T] \cup[T T A T T]
$$

is an ideal of $T$.

Corollary 6. If $A$ is a nonempty subset of a commutative ternary semigroup $T$ with identity, then

$$
\langle A\rangle=[\operatorname{TTTTA} .
$$

Proof. Consider:

$$
\begin{aligned}
\langle A\rangle & =A \cup[T T A] \cup[A T T] \cup[T A T] \cup[T T A T T] \\
& =A \cup[T T A] \cup[T T T T A] \\
& =[T T A] \cup[T T T T A] \\
& =[\text { TTTTA }] .
\end{aligned}
$$

Thus the assertion holds.

Definition 7. A proper ideal $A$ of a ternary semigroup $T$ is called a maximal ideal of $T$ if, for any ideal $B$ of $T, A \subseteq B \subseteq T$ implies $A=B$ or $B=T$.

Definition 8. ([2]) A proper ideal $P$ of a ternary semigroup $T$ is said to be prime if, for any ideal $A, B$ and $C$ of $T,[A B C] \subseteq P$ implies $A \subseteq P$ or $B \subseteq P$ or $C \subseteq P$.

The following was proved in [2]:

Theorem 9. A proper ideal $P$ of a commutative ternary semigroup $T$ is prime if, for all $x, y, z$ in $T$,

$$
[x y z] \in P \text { implies } x \in P \text { or } y \in P \text { or } z \in P \text {. }
$$

\section{Main Results}

We begin with the main result:

Theorem 10. Let $T$ be a commutative ternary semigroup with identity. If $M$ is a maximal ideal of $T$, then $M$ is prime. 
Proof. Assume that $M$ is a maximal ideal of $T$. To show that $M$ is prime, Theorem 9 allows us to show that, for all $x, y, z$ in $T,[x y z] \in M$ implies $x \in M$, $y \in M$ or $z \in M$. Let $x, y, z \in T$ be such that $[x y z] \in M$ and $x, y, z \notin M$. By Corollary 6 ,

$$
\langle M \cup\{x\}\rangle=[\operatorname{TTTT}(M \cup\{x\})] .
$$

Since $x \notin M$, we have

$$
M \subset M \cup\{x\} \subseteq[\operatorname{TTTT}(M \cup\{x\})] .
$$

Using the maximality of $M$, we get

$$
T=[\operatorname{TTTT}(M \cup\{x\})]
$$

Similarly, we obtain two conditions:

$$
T=[\operatorname{TTTT}(M \cup\{y\})]
$$

and

$$
T=[\operatorname{TTTT}(M \cup\{z\})] .
$$

Since $y \in T$ and the equation (3), there exist $a_{1}, b_{1}, c_{1}, d_{1} \in T$ and $u \in M \cup\{z\}$ such that $y=\left[a_{1} b_{1} c_{1} d_{1} u\right]$. If $u \in M$, then $y \in M$. This is a contradiction. Thus

$$
y=\left[a_{1} b_{1} c_{1} d_{1} z\right]
$$

Again, since $d_{1} \in T$ and the equation (2), there exist $a_{2}, b_{2}, c_{2}, d_{2} \in T$ and $v \in M \cup\{y\}$ such that $d_{1}=\left[a_{2} b_{2} c_{2} d_{2} v\right]$. If $v \in M$, then $y \in M$. This is a contradiction. Then $d_{1}=\left[a_{2} b_{2} c_{2} d_{2} y\right]$, and so

$$
\left.y=\left[a_{1} b_{1} c_{1} a_{2} b_{2} c_{2} d_{2} y\right] z\right]
$$

by the equation (4). Finally, since $d_{2} \in T$ and the equation (1), there exist $a_{3}, b_{3}, c_{3}, d_{3} \in T$ and $w \in M \cup\{x\}$ such that $d_{2}=\left[a_{3} b_{3} c_{3} d_{3} w\right]$. If $w \in M$, then $y \in M$. This is a contradiction. Then $d_{2}=\left[a_{3} b_{3} c_{3} d_{3} x\right]$, and hence

$$
y=\left[a_{1} b_{1} c_{1}\left[a_{2} b_{2} c_{2}\left[a_{3} b_{3} c_{3} d_{3} x\right] y\right] z\right]
$$

by (5). Since $[x y z] \in M, y \in M$. This is impossible. Thus $M$ is prime, and the proof completes.

Let $\left\{T_{i} \mid i \in I\right\}$ be an indexed family of ternary semigroups where 


$$
(x, y, z) \mapsto[x y z]_{i}
$$

is the ternary operation on $T_{i}$ for each $i \in I$. We define a ternary operation on the Cartesian product $\prod_{i \in I} T_{i}$ by

$$
\left[\left(x_{i}\right)_{i \in I},\left(y_{i}\right)_{i \in I},\left(z_{i}\right)_{i \in I}\right]=\left(\left[x_{i} y_{i} z_{i}\right]_{i}\right)_{i \in I}
$$

for all $\left(x_{i}\right)_{i \in I},\left(y_{i}\right)_{i \in I},\left(z_{i}\right)_{i \in I}$ in $\prod_{i \in I} T_{i}$. It is a routine matter to verify that $\prod_{i \in I} T_{i}$ is a ternary semigroup. If $I=\{1,2, \ldots, n\}$ we write $\prod_{i \in I} T_{i}$ as $T_{1} \times T_{2} \times$ $\cdots \times T_{3}$.

We need the following two lemmas to show that the converse of Theorem 10 is not valid in general.

Lemma 11. Let $\left\{T_{i} \mid i \in I\right\}$ be an indexed family of ternary semigroups such that $(x, y, z) \mapsto[x y z]_{i}$ is the ternary operation on $T_{i}$ for each $i \in I$. If $A_{i}$ is an ideal of $T_{i}$ for each $i \in I$, then $\prod_{i \in I} A_{i}$ is an ideal of $\prod_{i \in I} T_{i}$.

Proof. Since $A_{i} \neq \emptyset$ for all $i \in I$, we have $\prod_{i \in I} A_{i} \neq \emptyset$. If $\left(c_{i}\right)_{i \in I},\left(b_{i}\right)_{i \in I} \in$ $\prod_{i \in I} T_{i}$ and $\left(a_{i}\right)_{i \in I} \in \prod_{i \in I} A_{i}$, then

$$
\left[\left(b_{i}\right)_{i \in I}\left(a_{i}\right)_{i \in I}\left(c_{i}\right)_{i \in I}\right]=\left(\left[b_{i} a_{i} c_{i}\right]_{i}\right)_{i \in I} \in \prod_{i \in I} A_{i} .
$$

Similarly, we have

$$
\left[\prod_{i \in I} T_{i} \prod_{i \in I} T_{i} \prod_{i \in I} A_{i}\right] \subseteq \prod_{i \in I} A_{i}
$$

and

$$
\left[\prod_{i \in I} A_{i} \prod_{i \in I} T_{i} \prod_{i \in I} T_{i}\right] \subseteq \prod_{i \in I} A_{i} .
$$

Hence $\prod_{i \in I} A_{i}$ is an ideal of $\prod_{i \in I} T_{i}$ as required.

We now consider the closed interval of real numbers $T=[0,1]$. Under the ternary operation on $T$ defined by $(x, y, z) \mapsto x y z$, it is clear that $T$ is a ternary semigroup. The next lemma shows that, for any $a \in T,[0, a]$ is an ideal of $T$.

Lemma 12. For any $a \in T, A_{a}=[0, a]$ is an ideal of $T$.

Proof. Let $a \in T$, i.e., $a \in[0, a]$. Then $A_{a} \neq \emptyset$. If $x, y \in T$ and $z \in[0, a]$, then

$$
0 \leq x z y, x y z, z x y \leq a .
$$

Hence $A_{a}$ is an ideal of $T$.

The following example shows that the converse of the theorem is not true in general. 
Example 13. We consider the ternary semigroup

$$
T \times T=[0,1] \times[0,1] .
$$

Clearly, $T \times T$ is a commutative ternary semigroup with identity $(1,1)$. By Lemma $12, A_{0}=\{0\}$ is an ideal of $T$. Then by Lemma 11 we have

$$
A=[0,1] \times\{0\}
$$

is an ideal of $T \times T$.

To show that $A$ is prime we use Theorem 9 . Let $\left(x_{1}, y_{1}\right),\left(x_{2}, y_{2}\right),\left(x_{3}, y_{3}\right) \in$ $T \times T$ be such that $\left[\left(x_{1}, y_{1}\right)\left(x_{2}, y_{2}\right)\left(x_{3}, y_{3}\right)\right] \in A$; thus

$$
\left[x_{1} x_{2} x_{3}, y_{1} y_{2} y_{3}\right] \in A,
$$

and so $y_{1} y_{2} y_{3}=0$. This implies that $y_{1}=0, y_{2}=0$ or $y_{3}=0$. Therefore, $A$ is prime.

Since $A_{1 / 2}$ is an ideal of $T$, it follows that

$$
A \subset[0,1] \times A_{1 / 2} \subset T \times T,
$$

whence $A$ is not maximal.

\section{Acknowledgments}

The author was supported by the Faculty of Science, Khon Kaen University.

\section{References}

[1] I. N. Herstein, Topics in algebra, New York, Blaisdell Publishing Company, 1964.

[2] S. Kar, B. K. Maity, Some ideals of ternary semigroups, Analele Stiintifice Ale Universittii Matematica, LVII (2011), 247-258.

[3] S. Kar, B. K. Maity, Congruence on ternary semigroups, Journal of the Chungcheong Mathematical Society, 20 (2007), no. 3, 191-201.

[4] N. Kehayopulu, J. Ponizovskii, M. Tsingelis, A note on maximal ideals in ordered semigroups, Algebra and Discrete Mathematics, 2 (2003), 32-35. 
[5] D. H. Lehmer, A ternary analogue of abelian groups, Am. J. Math., 54 (1932), 329-338.

[6] M. L. Santiago, S. Sri Bala, Ternary semigroups, Semigroup Forum, 81 (2010), 380-388.

[7] F. M. Sioson, Ideal theory in ternary semigroups, Math. Jpn., 10 (1965), 63-84.

[8] Št. Schwarz, Prime ideals and maximal ideals in semigroups, Czechoslovak Mathematical Journal, 19 (1969), 72-79. 
